\title{
LINC01816 promotes the migration, invasion and epithelial-mesenchymal transition of thyroid carcinoma cells by sponging miR-34c-5p and regulating CRABP2 expression levels
}

\author{
HONGYUAN ZHAO ${ }^{1}$, XIAOFENG ZHU ${ }^{2}$, YI LUO ${ }^{2}$, SHENGSHAN LIU ${ }^{2}$, \\ WENSHUANG WU ${ }^{1}$, LINGYUN ZHANG ${ }^{1}$ and JINGQIANG ZHU ${ }^{1}$ \\ ${ }^{1}$ Department of Thyroid and Parathyroid Surgery Center, West China Hospital, Sichuan University, \\ Chengdu, Sichuan 610000; ${ }^{2}$ Department of Thyroid and Breast Surgery, \\ Affiliated Hospital of Zunyi Medical University, Zunyi, Guizhou 510515, P.R. China
}

Received July 25, 2020; Accepted February 1, 2021

DOI: 10.3892/or.2021.8032

\begin{abstract}
Thyroid carcinoma (THCA) is a common type of endocrine system cancer and its current clinical treatment method is surgical resection. Long non-coding RNAs (lncRNAs) have been revealed to serve important roles in a variety of complex human diseases. Therefore, determining the association between IncRNAs and diseases may provide novel insight into disease-related lncRNAs, with the aim of improving disease treatments and diagnoses. Long intergenic non-protein coding RNA 1816 (LINC01816) was identified to be associated with the survival of patients with colorectal cancer using the IDHI-MIRW method. The present study aimed to investigate the role and molecular mechanism of LINC01816 in THCA. Analysis of datasets from The Cancer Genome Atlas database revealed that the upregulation of LINC01816 expression levels was associated with a variety of cancer types. Reverse transcription-quantitative PCR analysis demonstrated that compared with the normal thyroid tissues, the expression levels of LINC01816 were upregulated in THCA tissues. The results of wound healing and Transwell assays, and western blotting demonstrated that the overexpression of LINC01816 could strengthen the invasive and migratory abilities of THCA cells and enhance epithelial-mesenchymal transition progression. Analysis using the starBase website and dual-luciferase reporter assays identified that microRNA (miR)-34c-5p was a target of LINC01816. The overexpression of miR-34c-5p could inhibit the invasive and migratory abilities of THCA
\end{abstract}

Correspondence to: Dr Jingqiang Zhu, Department of Thyroid and Parathyroid Surgery Center, West China Hospital, Sichuan University, 37 Guoxue Lane, Wuhou, Chengdu, Sichuan 610000, P.R. China

E-mail: zjq-wkys@163.com

Key words: thyroid carcinoma, long intergenic non-protein coding RNA 1816, microRNA-34c-5p, cellular retinoic acid binding protein 2 , invasion, metastasis cells, in addition to inhibiting the cellular retinoic acid binding protein 2 (CRABP2) overexpression-induced effects on invasion, migration and EMT processes. In conclusion, the findings of the present study indicated that LINC01816 may be capable of sponging miR-34c-5p to upregulate CRABP2 expression levels, which subsequently promoted the invasion, migration and EMT of THCA cells. Therefore, targeting the LINC01816/miR-34c-5p/CRABP2 pathway may be an effective therapeutic approach for patients with THCA.

\section{Introduction}

Thyroid carcinoma (THCA) is the most common type of endocrine system malignancy and accounts for $\sim 1 \%$ of all malignancies (1). The incidence of THCA has continued to increase worldwide over the past few decades $(2,3)$, especially in women in China and the United States $(4,5)$. THCA originates from thyroid follicular or parafollicular cells and is usually classified into differentiated THCA (follicular THCA and papillary THCA), medullary THCA and undifferentiated THCA (6). The majority of THCAs exhibit relatively low levels of malignancy, except for undifferentiated THCA $(7,8)$. Most patients with differentiated THCA have a favorable prognosis and rarely exhibit signs of distant metastasis. Only 1-3\% of THCAs are classified as undifferentiated THCAs $(7,8)$; however, the mortality rate of undifferentiated THCA is $14-50 \%$ and the average survival time of patients with undifferentiated THCA is 3-5 months (8). The current prognosis of patients with undifferentiated THCA is poor due to the rapid onset and aggressiveness of the tumor, and the occurrence of widespread pelvic metastasis (9). Thus, further studies investigating the molecular mechanisms of invasion and metastasis in undifferentiated THCA are urgently required.

Only $1-2 \%$ of the human genome transcript encodes proteins, whereas $>75 \%$ is transcribed into non-coding RNA (ncRNA) (10). There are numerous types of ncRNA, including long ncRNAs (lncRNAs), which are $>200$ nucleotides in length, and microRNAs (miRNAs/miRs) (11). Both lncRNAs and miRNAs have been reported to be involved in the invasion and metastasis of tumor cells. For instance, the lncRNA 
colorectal neoplasia differentially expressed was discovered to promote glioma cell growth and invasion through the mTOR signaling pathway (12). The IncRNA, H19, a host gene of miR-675, was revealed to generate mature miR-675-3p and miR-675-5p, which were subsequently demonstrated to be involved in tumor metastasis (13). On the other hand, miRNAs, which are 20-25 nucleotides in length, regulate the expression of specific target genes that have roles in numerous biological processes, such as tumor progression and survival (14-17). Previous studies have reported that miRNAs regulated proliferation and invasion in THCA. For instance, Dong et al revealed that the knockdown of miR-363-3p expression in papillary THCA inhibited tumor progression by targeting NIN1 (RPN12) binding protein 1 homolog (18); and miR-219 was revealed to inhibit cell viability and metastasis in papillary THCA by targeting EYA transcriptional coactivator and phosphatase 2 (19). miRNAs have also been revealed to play crucial roles in numerous tumor processes. Previous evidence suggested that miR-34c-5p expression was closely associated with a poor prognosis and unfavorable clinicopathological parameters $(20,21)$. Shen et al indicated that the IncRNA KCNQ1 opposite strand/antisense transcript 1 sponged miR-34c-5p to promote osteosarcoma growth via aldolase, fructose-bisphosphate A-enhanced aerobic glycolysis (22). Numerous other studies have revealed that miR-34c-5p was closely related to the development of numerous types of human malignant cancer (23-25).

The functions of lncRNAs and miRNAs do not occur in isolation. In fact, it has been well established that a regulatory network composed of lncRNAs and miRNAs has an enhanced effect on the regulation of cellular processes (26). miRNAs often inhibit protein translation through binding to the 3'-untranslated region (UTR) of target mRNAs, and lncRNAs can sponge miRNAs and repress this process (27). It has been reported that lncRNA CCDC144NL antisense RNA 1 sponged miR-143-3p and regulated mitogen-activated protein kinase kinase kinase 7 by acting as a competing endogenous RNA (ceRNA) in gastric cancer (28).

In general, treatment strategies for cancer are divided into three major categories: Surgical resection, chemotherapy and radiotherapy (29). Surgical resection is the primary treatment option recommended for non-metastatic tumors. Metastatic cancers are treated with combined therapies; however, these therapeutic regimens are often insufficient to effectively treat metastatic tumors currently (30). Therefore, there is an urgent requirement to develop effective and novel approaches for the treatment of cancer. Muhammad et al previously reported that bitter melon extract inhibited breast cancer growth in a preclinical model by inducing autophagic cell death (31). Cholesterol depletion by methyl- $\beta$-cyclodextrin was demonstrated to increase tamoxifen-induced cell death by enhancing its uptake in melanoma (32). In addition, methyl- $\beta$-cyclodextrin was revealed to enhance the sensitivity of skin cancer cells to doxorubicin by regulating wild-type (Wt) p53 (33). Furthermore, numerous drugs have been designed to treat cancer by targeting tumor-related targets. In a previous study, cellular retinoic acid (RA) binding protein 2 (CRABP2) was discovered to be an essential protein for tumor growth. The abnormal expression of CRABP2 was revealed to be associated with malignant human cancer types. In addition, the knockdown of CRABP2 expression levels was revealed to suppress the migration of cancer cells (34-36). Previous studies on cancer cells have also revealed that the delivery of RA by CRABP2 facilitated RAR-related orphan receptor A transcriptional activity by enhancing differentiation and apoptosis, and reducing proliferation $(37,38)$.

The present study used datasets from The Cancer Genome Atlas (TCGA) database to reveal that the relative expression levels of LINC01816 were upregulated in patients with THCA compared with healthy patients. The lncRNA long intergenic non-protein coding RNA 1816 (LINC01816) was also revealed to promote epithelial-mesenchymal transition (EMT), invasion and metastasis in THCA tissues. Bioinformatics analysis subsequently identified binding sites between miR-34c-5p and LINC01816 or the 3'-UTR of CRABP2, which indicated the presence of a competing endogenous RNA (ceRNA) regulatory mechanism. These findings suggested that ncRNAs have the potential to become a novel research hotspot in the context of cancer therapy.

\section{Materials and methods}

Patient samples. Matched paracarcinoma and cancer tissues were obtained from 10 patients (Table S1) undergoing surgery for THCA at The Affiliated Hospital of Zunyi Medical University (Zunyi, China). The present study was approved by the Zunyi Medical University Ethics Committee and informed patient consent was obtained prior to participation.

Cell lines and culture. Human THCA cell lines, OCUT-2C, C643, 8305C and CAL-62, as well as the normal thyroid cell line, HTori-3 were purchased from the Institute of Cell Biology, Chinese Academy of Sciences (Shanghai, China). OCUT-2C, C643, 8305C and CAL-62 were cultured in DMEM supplemented with $1 \%$ antibiotics $(100 \mathrm{U} / \mathrm{ml}$ penicillin and $100 \mu \mathrm{g} / \mathrm{ml}$ streptomycin sulfates) and 10\% FBS (all from Gibco; Thermo Fisher Scientific, Inc.). HTori-3 was maintained in DMEM/F12 supplemented with $1 \%$ glutamine and $10 \%$ FBS. Cells were maintained in an incubator at $37^{\circ} \mathrm{C}$ in a humidified atmosphere with $5 \% \mathrm{CO}_{2}$. In all experiments, cells were allowed to acclimatize for $24 \mathrm{~h}$ prior to treatment. Cell lines were tested and confirmed to be mycoplasma-free at the beginning and end of the research.

Cell transfection. Cells were transfected upon reaching $60-70 \%$ confluence with pCDNA3.1-LINC01816 or pCDNA3.1-CRABP2 plasmids, miR-34c-5p mimics, miR-34c-5p inhibitor and their corresponding controls using Lipofectamine $^{\circledR} 3000$ reagent (Invitrogen; Thermo Fisher Scientific, Inc.), according to the manufacturer's protocol. The miR-34c-5p mimics sequence was 5'-AGGCAGUGU AGUUAGCUGAUUGC-3' and the sequence of the mimics control was 5'-UUACUCGACACGUGUCAAGUUU-3'. The miR-34c-5p inhibitor sequence was 5'-GCAAUCAGCUAA CUACACUGCCU-3' and the sequence of inhibitor control was 5'-CAGUACUUUUGUGUAGUACAA-3' The C643 or OCUT-2C cells $\left(2 \times 10^{5}\right.$ cells/dish) were seeded into a $6-\mathrm{cm}$ cell culture dish and cultured to $60-70 \%$ confluence. The cells were transfected with $1 \mu \mathrm{g}$ plasmids or $30 \mathrm{nM}$ miRNA mimics/inhibitor in the $6-\mathrm{cm}$ cell culture dish. The transfected 
cells were incubated at $37^{\circ} \mathrm{C}$ for $48 \mathrm{~h}$. The transfected cells were harvested $48 \mathrm{~h}$ post transfection and used for subsequent experiments.

Scratch wound healing assay. A wound healing assay was performed using C643 and OCUT-2C cells to determine the levels of cell migration. Briefly, cells $\left(2 \times 10^{6}\right.$ cells/well) were seeded into 24 -well plates and cultured to $100 \%$ confluence. Then, a linear scratch was made in the cell monolayer using a sterile 200- $\mu$ l pipette tip. The scratch area was imaged at 0 and $24 \mathrm{~h}$ using an optical microscope (Olympus Corporation). The scratch width was captured at a magnification of $x 100$. The area of each scratch wound was determined using ImageJ software (v1.48; National Institutes of Health). The cell migratory rate was calculated as: Migration rate $(\%)=($ initial distance $-24 \mathrm{~h}$ scratch distance) $\mathrm{x} 100$.

Cell invasion assay. The cell invasion assay was performed using a Matrigel invasion chamber (Corning, Inc.) in a 24-well Transwell plate ( $8 \mu \mathrm{m}$ pore size). Briefly, the cell density was adjusted to $5 \times 10^{5}$ cells $/ \mathrm{ml}$. Then, $500 \mu \mathrm{l}$ of serum-free medium containing $3 \times 10^{4}$ cells was added into the upper chamber of the Transwell plate. A volume of $750 \mu 1$ medium supplemented with $10 \%$ FBS was added into the lower chamber. Following incubation at $37^{\circ} \mathrm{C}$ for $24 \mathrm{~h}$, the invasive cells in the lower chamber were fixed with $4 \%$ paraformaldehyde at room temperature for $20 \mathrm{~min}$ and subsequently stained with $0.5 \%$ crystal violet at $37^{\circ} \mathrm{C}$ for $30 \mathrm{~min}$; a cotton swab was used to gently remove the cells on the upper surface of the upper chamber. Stained cells were visualized using a light microscope (magnification, x100) in five randomly selected fields of view from each group.

$R N A$ extraction and reverse transcription-quantitative $P C R$ $(R T-q P C R)$. Total RNA was extracted from tissues or cultured cells using TRIzol ${ }^{\circledR}$ reagent (Invitrogen; Thermo Fisher Scientific, Inc.) for mRNA or a miRNeasy Micro kit (for miRNA; Qiagen $\mathrm{GmbH}$ ) for miRNA, according to the manufacturers' protocols. Then, $1 \mu \mathrm{g}$ RNA was reverse transcribed into cDNA using a Prime-Script RT-qPCR kit (Takara Bio, Inc.) for mRNA or a miScript II RT kit (Qiagen $\mathrm{GmbH}$ ) for miRNA, according to the manufacturer's protocol. The primer sequences used were as follows: LINC01816 forward, 5'-CAG CTGTCTTTGTCTGGGGCGGCGG-3' and reverse, 5'-GCC CCAGACAAAGACAGC-3'; miR-34c-5p forward, 5'-GAG GCAGTGTAGTTAGCTGA-3' and reverse, 5'-TCCAGTTTT TTTTTTTTTTTGCAATC-3'; CRABP2 forward, 5'-ATG CCCAACTTCTCTGGCAACTGGA-3' and reverse, 5'-CAG CATCACATTCACCC-3'; U6 forward, 5'-GTGCAGGGT CCGAGGT-3' and reverse, 5'-CTCGCTTCGGCAGCACA-3'; GAPDH forward, 5'-GGAGCGAGATCCCTCCAAAAT-3' and reverse, 5'-GGCTGTTGTCATACTTCTCATGG-3'. RT-qPCR was subsequently performed using a SYBR-Green PCR kit (Takara Bio, Inc.) on a LineGene 9600 Plus Real-Time PCR Detection system (Hangzhou Bioer Co., Ltd.). Thermal cycler conditions were as follows: 1 cycle at $98^{\circ} \mathrm{C}$ for $3 \mathrm{~min}$, 40 cycles at $98^{\circ} \mathrm{C}$ for $1 \mathrm{~min}, 60^{\circ} \mathrm{C}$ for $30 \mathrm{sec}$, and $72^{\circ} \mathrm{C}$ for $30 \mathrm{sec}, 1 \mathrm{cycle}$ of $72^{\circ} \mathrm{C}$ for $5 \mathrm{~min}$. The relative expression levels of mRNAs and miRNAs were calculated using the $2^{-\Delta \Delta \mathrm{Cq}}$ method (39). mRNA expression levels were normalized to
GAPDH expression levels and miRNA expression levels to U6 expression levels.

Dual-luciferase reporter assay. Cells were plated into 6-cm culture dishes at a density of $5 \times 10^{5}$ cells $/ \mathrm{ml}$. The Wt or mutant (Mut) 3'-UTR sequences of CRABP2 and LINC01816 were cloned into a psiCHECK2 luciferase reporter plasmid (Promega Corporation) containing Renilla and firefly luciferase. The luciferase reporter vectors and miR-34c-5p were co-transfected into C643 or OCUT-2C cells using Lipofectamine 3000, according to the manufacturer's protocol. Following $24 \mathrm{~h}$ of transfection, cells were lysed with $1 \mathrm{X}$ lysis buffer (Beyotime Institute of Biotechnology) and the relative luciferase activity was measured using a Dual-Luciferase Reporter assay system (Beyotime Institute of Biotechnology). Firefly luciferase activity was normalized to Renilla luciferase activity.

RNA immunoprecipitation (RIP) assay. RIP was performed using an RNA Binding Protein Immunoprecipitation kit (EMD Millipore), according to the manufacturer's protocol. Briefly, C643 and OCUT-2C cells were digested and resuspended in PBS ( $1 \times 10^{7}$ cells in $2 \mathrm{ml}$ PBS). The RIP assay was performed using an anti-AGO2 antibody (1:500; product code ab32381; Abcam) overnight at $4^{\circ} \mathrm{C}$ and an anti-IgG antibody (1:500; product no. 3900S; Cell Signaling Technology, Inc.) as the negative control. RT-qPCR was subsequently performed to analyze the CRABP2 mRNA expression levels.

Immunofluorescence staining. Cells were seeded into $6-\mathrm{cm}$ dishes at a density of $2 \times 10^{5}$ cells/dish. The cells were fixed with $4 \%$ paraformaldehyde for $20 \mathrm{~min}$ at room temperature, permeabilized in $0.5 \%$ Triton $\mathrm{X}-100$ for $1 \mathrm{~h}$ and blocked with $3 \%$ BSA at room temperature for $2 \mathrm{~h}$ (Nanjing KeyGen Biotech Co., Ltd.). Subsequently, the cells were incubated with the following primary antibodies for $6 \mathrm{~h}$ at $4^{\circ} \mathrm{C}$ in humid conditions: Anti-E-cadherin (1:100; product no. 14472S; Cell Signaling Technology, Inc.), anti-vimentin (1:100; product no. 5741S; Cell Signaling Technology, Inc.) and anti-CRABP2 (1:250; product code ab211927; Abcam). Following the primary antibody incubation, the cells were incubated with Alexa Fluor-594 anti-rabbit (1:500; cat. no. A-11012; Thermo Fisher Scientific, Inc.) or anti-mouse secondary antibodies (1:500; cat. no. A-11032; Thermo Fisher Scientific, Inc.) at room temperature for $1 \mathrm{~h}$. The cell nuclei were subsequently stained with $5 \mu \mathrm{g} / \mathrm{ml}$ DAPI (Beyotime Institute for Biotechnology) for $5 \mathrm{~min}$. Stained cells were visualized using an immunofluorescence microscope at a magnification of x400 (Olympus Corporation). Western blotting. Total protein was extracted from cells using RIPA lysis buffer (product no. P0013C; Beyotime Institute of Biotechnology) supplemented with protease and phosphatase inhibitors. Total protein concentration was quantified using a BCA protein assay kit (Beyotime Institute of Biotechnology) and $20 \mu \mathrm{g}$ protein per lane was separated via $10 \%$ SDS-PAGE. The separated proteins were subsequently transferred onto PVDF membranes (EMD Millipore) and blocked with 5\% BSA at $37^{\circ} \mathrm{C}$ for $1 \mathrm{~h}$. The membranes were then incubated overnight at $4^{\circ} \mathrm{C}$ with the following primary antibodies: Anti-CRABP2 (1:1,000; product code ab211927; Abcam), anti-E-cadherin (1:1,000; product no. 14472S; Cell Signaling Technology, Inc.), 
anti-N-cadherin $(1: 1,000$; product no. $13116 \mathrm{~S}$; Cell Signaling Technology, Inc.), anti-vimentin (1:1,000; product no. 5741S; Cell Signaling Technology, Inc.), anti-NME1 (1:1,000; cat. no. 11086-2-AP; ProteinTech Group, Inc.), anti-AMF (1:1,000; product code ab66340; Abcam), anti-cyclin D1 (1:1,000; product no. 55506S), anti-cyclin E1 (1:1,000; product no. 20808S), p27 (1:1,000; product no. 3686S), p21 (1:1,000; product no. 2947S), cleaved caspase-3 (1:1,000; product no. 9664S), cleaved PARP (1:1,000; product no. $5625 \mathrm{~S})$, Bax (1:1,000; product no. 5023S) and Bcl-2 (1:1,000; product no. 15071S; all from Cell Signaling Technology, Inc.) and anti-GAPDH (1:10,000; cat. no. 60004-1-AP; ProteinTech Group, Inc.). Following the primary antibody incubation, the PVDF membranes were washed 3 times with TBS-Tween-20 $(0.05 \%$; $5 \mathrm{ml}$ Tween-20 in 1,000 $\mathrm{ml}$ TBS) and then incubated for an additional $2 \mathrm{~h}$ with a horseradish peroxidase-conjugated secondary antibody (1:5,000; cat. no. BA1056; Wuhan Boster Biological Technology, Ltd.) at room temperature for 2 h. Protein bands were visualized using an ECL Western Blotting Detection kit (Beyotime Institute of Biotechnology). Densitometric analysis was performed using ImageJ software (v1.48; National Institutes of Health). Protein expression levels were normalized to GAPDH expression levels.

Cell cycle analysis. A Cell Cycle kit (Beyotime, China) was used for cell cycle analysis. Cells $\left(1 \times 10^{6}\right.$ cells $\left./ \mathrm{ml}\right)$ were washed three time by cold PBS and then fixed in $70 \%$ ethanol at $-20^{\circ} \mathrm{C}$ for $24 \mathrm{~h}$. Subsequently, cells were washed with cold PBS and stained with appropriate propidium iodide (PI) staining buffer containing $10 \%$ RNase A at $37^{\circ} \mathrm{C}$ for 30 min in the dark. Cell cycle analysis was performed using FACSCantoII cytometer (BD Biosciences). Analyses were performed on Modifit LT 4.1 software (BD Biosciences).

Bioinformatics analysis. The expression levels of LINC01816 in different types of cancer were obtained from the online GEPIA database (40) (http://gepia.cancer-pku.cn/). The target predictions between LINC01816/miR-34c-5p were analyzed using the online starBase v2.0 database (41) (http://starbase. sysu.edu.cn). The corresponding target genes for miR-34c-5p were filtered out using the TargetScan database (42) (https://www.targetscan.org) and the KEGG pathway analysis was performed using the Database for Annotation, Visualization and Integrated Discovery (DAVID) database (43) (https://david-d.ncifcrf.gov/).

Statistical analysis. Statistical analysis was performed using GraphPad Prism 8.0 software (GraphPad Software, Inc.) and SPSS 22.0 software (IBM Corp.). All data are presented as the mean \pm SD of $\geq 3$ experimental repeats. Statistical differences between groups were analyzed using unpaired Student's t-tests. $\mathrm{P}<0.05$ was considered to indicate a statistically significant difference.

\section{Results}

LINC01816 expression levels are upregulated in undifferentiated THCA tissues and cell lines. Analysis of THCA RNA sequencing datasets from TCGA revealed that LINC01816 expression levels were upregulated in THCA tissues compared with normal tissues (Fig. 1A). Thus, the expression levels of LINC01816 in THCA and matched adjacent paracarcinoma thyroid tissues obtained from 10 patients were analyzed. RT-qPCR analysis of the 10 paired thyroid tissues revealed that the expression levels of LINC01816 were upregulated in cancerous tissues compared with noncancerous tissues (Fig. 1B and C). Moreover, the RT-qPCR results indicated that the expression levels of LINC01816 were significantly upregulated in the undifferentiated THCA cell lines, C643, 8305C and CAL-62, compared with the normal thyroid cell line, OCUT-2C (Fig. 1D).

LINC01816 promotes the EMT, migration and invasion of undifferentiated THCA cells. To investigate the role of LINC01816 in undifferentiated THCA, pcDNA3.1-LINC01816 plasmids were transfected into C643 and OCUT-2C cells to promote the overexpression of LINC01816 (Fig. 2A). Transwell assays were used to detect the cell invasive ability. As revealed in Fig. 2B, the number of invasive cells was increased in the LINC01816 overexpression group compared with the vector group. The results of the wound healing assay also demonstrated that the migratory rate was increased in cells overexpressing LINC01816 compared with the vector group (Fig. 2C). Immunofluorescence staining revealed that E-cadherin was downregulated while vimentin was upregulated after LINC01816 overexpression in C643 and OCUT-2C cells (Fig. 2D). In addition, the protein expression levels of E-cadherin were downregulated in the LINC01816 overexpression group, while the expression levels of $\mathrm{N}$-cadherin and vimentin were upregulated in the LINC01816 overexpression group (Fig. 2E). Furthermore, the expression levels of NME1 were also downregulated following the overexpression of LINC01818, while the expression levels of AMF were upregulated, in C643 and OCUT-2C cells (Fig. 2F).

To determine the effects of LINC01818 on the invasion and migration, cell Transwell and scratch wound healing assay were performed in Htori-3 cells. LINC01816 promoted cell invasion and migration in normal thyroid cells (Fig. S1). To determine the effects of LINC01818 on the EMT process, LINC01818 expression was knocked down in C643 cells. Following the knockdown of LINC01818, the expression levels of E-cadherin were upregulated, while the expression levels of N-cadherin and vimentin were downregulated (Fig. S2A). Similarly, the results of the Transwell invasion and wound healing assays indicated that the knockdown of LINC01816 expression suppressed cell invasion and migration in C643 cells (Fig. S2B and C). In addition, the cell cycle distribution was detected using flow cytometry. The overexpression of LINC01816 resulted in $\mathrm{G}_{2} / \mathrm{M}$ phase arrest (Fig. S3A). Western blotting was performed to analyze the expression levels of cell cycle-related proteins, including cyclin D1, cyclin E1, p27 and p21. The expression levels of cyclin D1 and cyclin E were downregulated following the overexpression of LINC01816, while the expression levels of p27 and p21 were upregulated (Fig. S3B). Thus, it was suggested that the cell cycle arrest in the $G_{2} / M$ phase may promote EMT. The expression levels of the apoptosis-related proteins, cleaved caspase-3 (c-caspase-3), cleaved poly (ADP-ribose) polymerase (c-PARP), Bax and Bcl-2, were also analyzed using western blotting. These results revealed that the changes in LINC01816 expression had 

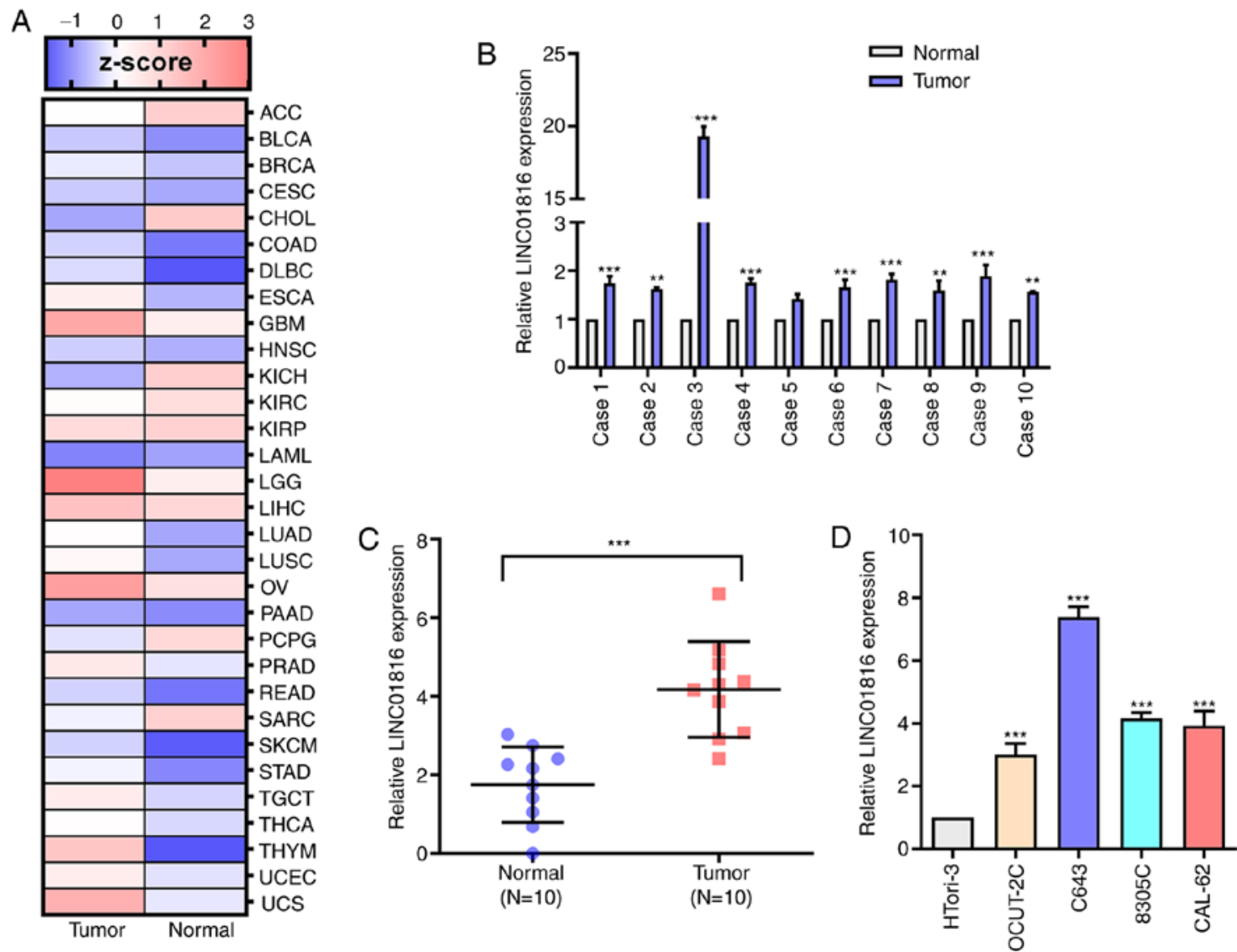

Figure 1. The expression of LINC01816 is detected in a validated cohort of patients with thyroid carcinoma and in thyroid carcinoma cell lines. (A) The LINC01816 expression in various human cancers according to The Cancer Genome Atlas database. (B) The expression of LINC01816 in cancer tissue of patients with thyroid carcinoma was significantly higher than that in normal tissue. (C) The statistics of LINC01816 expression in the tumor group (N=10) and normal group ( $\mathrm{N}=10)$. (D) The expression of LINC01816 in various thyroid cancer cells and normal thyroid cells. All of the aforementioned experiments were repeated at least three times. ${ }^{* * *} \mathrm{P}<0.01$ and ${ }^{* * *} \mathrm{P}<0.001$. LINC01816, long intergenic non-protein coding RNA 1816.

little effect on the levels of cell apoptosis in vitro (Fig. S3C). These findings aforementioned indicated that the upregulated expression levels of LINC01816 may induce EMT, leading to an increase in cell invasion.

miR-34c-5p is regulated by LINC01816 and inhibits EMT and cell migration. The bioinformatics online tool, starBase, was used to predict that LINC01816 may act as a sponge by binding to $\mathrm{miR}-34 \mathrm{c}-5 \mathrm{p}$ at the binding site located within chromosome 2: 70351393-70351399[-] (Fig. 3A). Following the overexpression of LINC01816, the expression levels of miR-34c-5p were demonstrated to be downregulated in C643 and OCUT-2C cells (Fig. 3B). Moreover, the results of the dual-luciferase reporter assay revealed that the overexpression of miR-34c-5p reduced the relative luciferase activity of LINC01816-Wt vectors, while no effect was observed on the relative luciferase activity of LINC01816-Mut vectors (Fig. 3C). The RIP assay results also revealed that LINC01816 and miR-34c-5p were markedly enriched in anti-Ago2 lysates, which suggested that LINC01816 may sponge miR-34c-5p (Fig. 3D). As revealed in Fig. 3E and F, the results of the wound healing assay and Transwell invasion assay revealed that the migratory rate and number of invasive cells were decreased in cells overexpressing miR-34c-5p compared with the mimics NC group. To determine whether miR-34c-5p inhibited EMT in THCA, the protein expression levels of E-cadherin, $\mathrm{N}$-cadherin and vimentin were analyzed by western blotting following the inhibition of miR-34c-5p in C643 cells. The results revealed that the expression levels of E-cadherin were downregulated, while the expression levels of $\mathrm{N}$-cadherin and vimentin were upregulated following the inhibition of miR-34c-5p (Fig. S2A). Transwell and wound healing assays were also performed and the results demonstrated that the inhibition of miR-34c-5p expression promoted cell invasion and migration in C643 cells (Fig. S2B and C). Conversely, the expression levels of E-cadherin were significantly upregulated, while vimentin and $\mathrm{N}$-cadherin expression levels were downregulated in the miR-34c-5p mimics group (Fig. 3G). Immunofluorescence staining revealed that $\mathrm{E}$-cadherin was upregulated while vimentin was downregulated after miR-34c-5p overexpression in C643 and OCUT-2C cells (Fig. 3H). These data indicated that miR-34c-5p may attenuate the migratory and invasive abilities, and the EMT of THCA cells, via sponging LINC01816.

$C R A B P 2$ is a target gene of miR-34c-5p and is associated with $E M T$, migration and invasion. Immunohistochemistry was used to analyze the expression levels of the CRABP2 protein in THCA and paracarcinoma tissues of patients. As revealed in Fig. 4A, CRABP2 protein expression levels were significantly upregulated in THCA tissues compared with paracarcinoma 

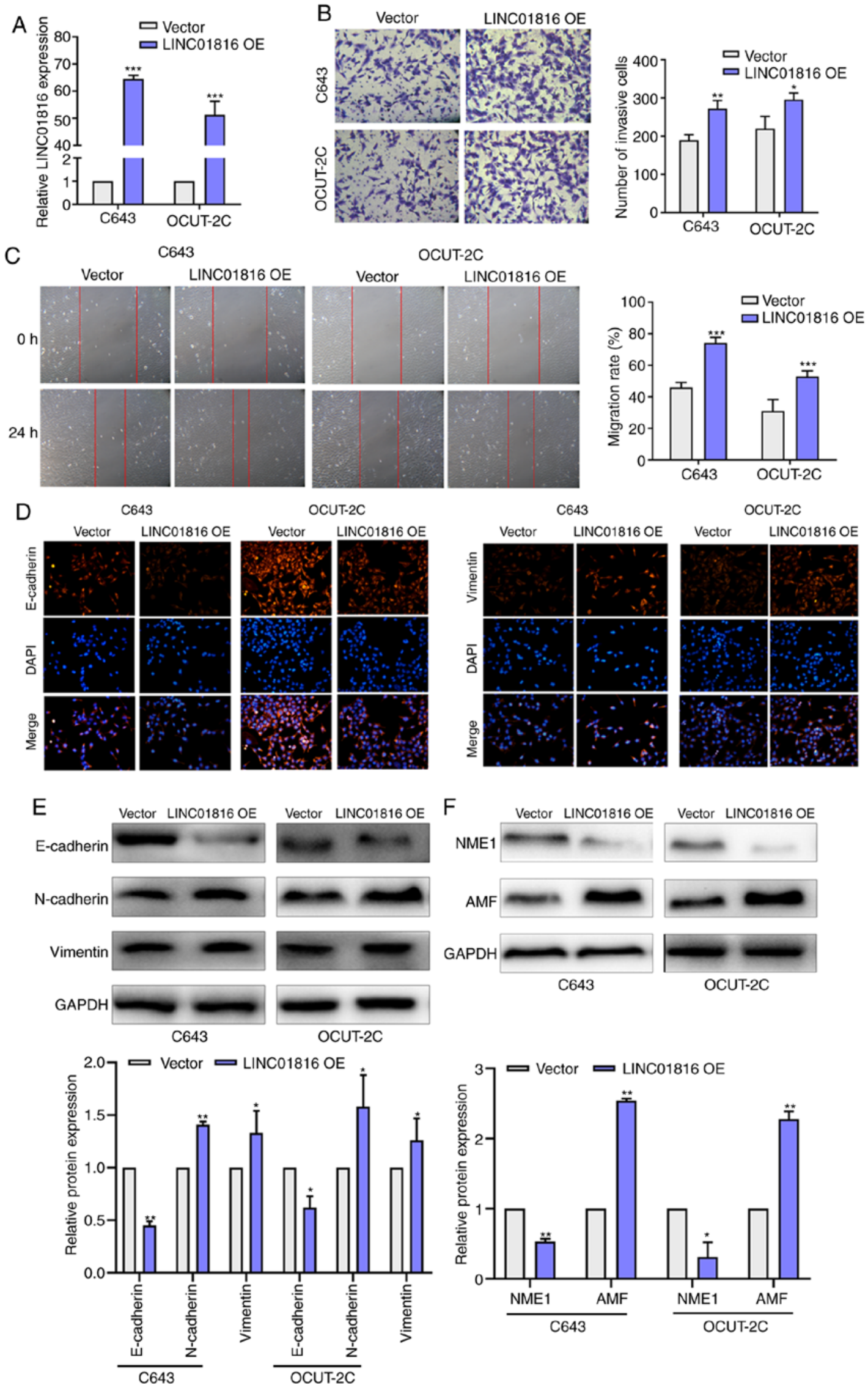

Figure 2. LINC01816 promotes the migration and EMT of thyroid carcinoma cells. (A) Validation of cell transfection efficiency. (B) Transwell assay was performed to assess the invasive ability of C643 and OCUT-2C cells with LINC01816 overexpression. (C) Scratch wound healing assay was performed to assess the migration ability of C643 and OCUT-2C cells with LINC01816 overexpression. (D) Immunofluorescence staining was used to detect E-cadherin and vimentin expression after LINC01816 overexpression in C643 and OCUT-2C cells. (E and F) The EMT and migration-related proteins were detected after LINC01816 overexpression in C643 and OCUT-2C cells. All of the aforementioned experiments were repeated at least three times. ${ }^{*} \mathrm{P}<0.05$, ${ }^{* * *} \mathrm{P}<0.01$ and ${ }^{* * *} \mathrm{P}<0.001$. LINC01816, long intergenic non-protein coding RNA 1816; EMT, epithelial-mesenchymal transition; OE, overexpression. 
A
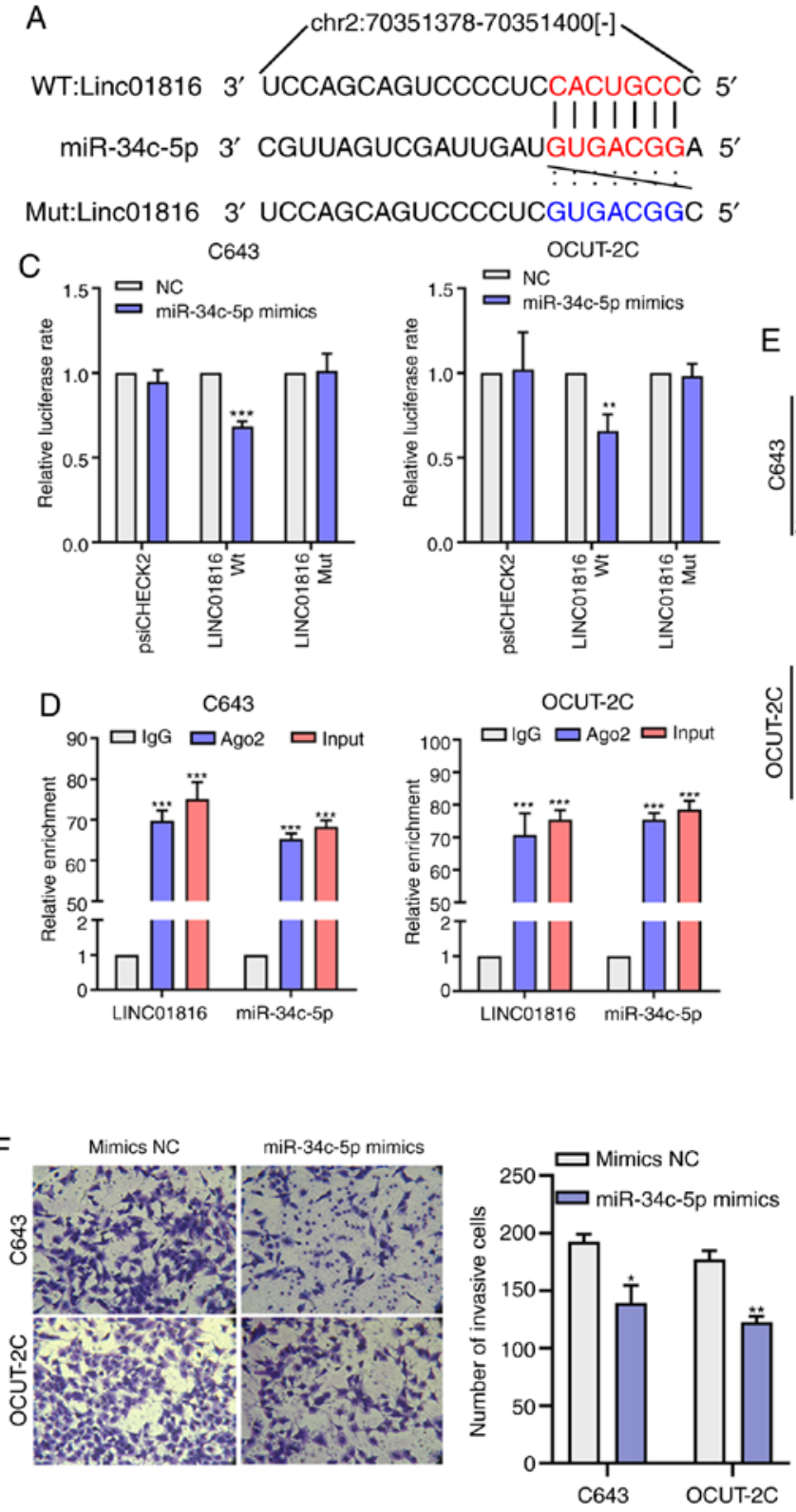

$\stackrel{\substack{5 \\ \vdots}}{0 \mathrm{~h}}$
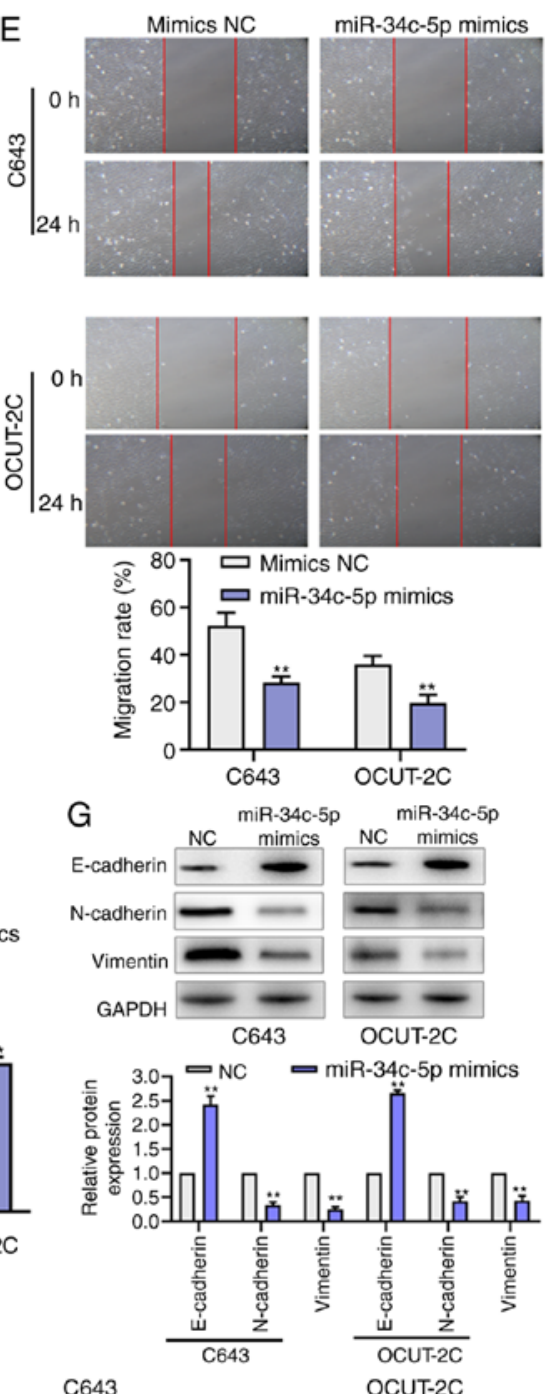

$\mathrm{H}$

C643

OCUT-2C
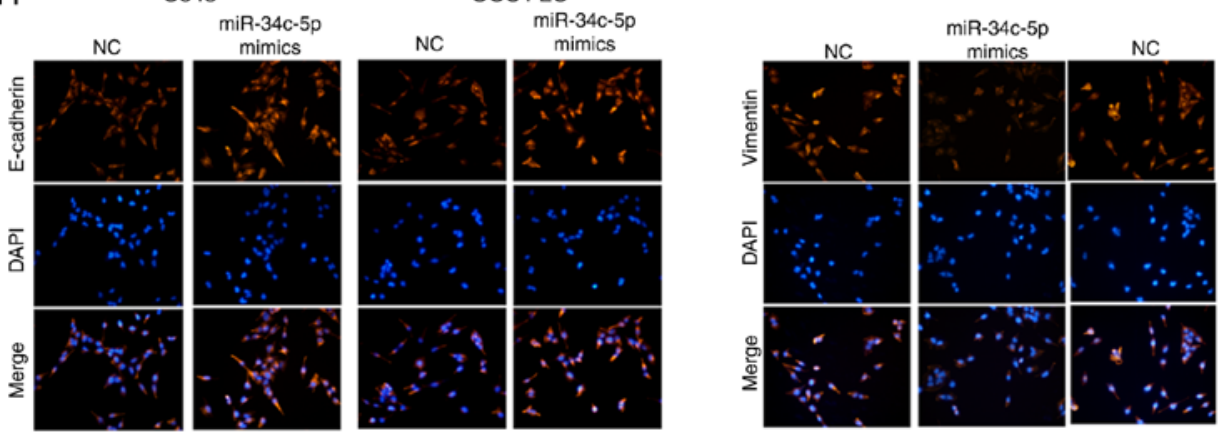

miR-34c-5p

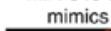

Figure 3. LINC01816 acts as a sponge of miR-34c-5p. (A) Schematic diagram of the miR-34c-5p putative binding sites and mutant site in LINC01816. (B) The miR-34c-5p expression was detected after LINC01816 overexpression in C643 and OCUT-2C cells. (C) Dual luciferase reporter gene assay revealed that miR-34c-5p significantly decreased the luciferase activity of LINC01816. (D) RNA immunoprecipitation assay of the enrichment of Ago2 on LINC01816 relative to IgG in C643 and OCUT-2C cells. (E) Scratch wound healing assay was performed to assess the migration ability of C643 and OCUT-2C cells with miR-34c-5p overexpression. (F) Transwell assay was performed to assess the invasive ability of C643 and OCUT-2C cells with miR-34c-5p overexpression. (G) The EMT-related proteins were detected after miR-34c-5p overexpression in C643 and OCUT-2C cells. (H) Immunofluorescence staining was used to detect E-cadherin and vimentin expression after miR-34c-5p overexpression in C643 and OCUT-2C cells. All of the aforementioned experiments were repeated at least three times. ${ }^{*} \mathrm{P}<0.05,{ }^{* * *} \mathrm{P}<0.01$ and ${ }^{* * *} \mathrm{P}<0.001$. LINC01816, long intergenic non-protein coding RNA 1816; miR-34c-5p, microRNA-34c-5p; EMT, epithelial-mesenchymal transition; Wt, wild-type; Mut, mutant; OE, overexpression; NC, negative control. 

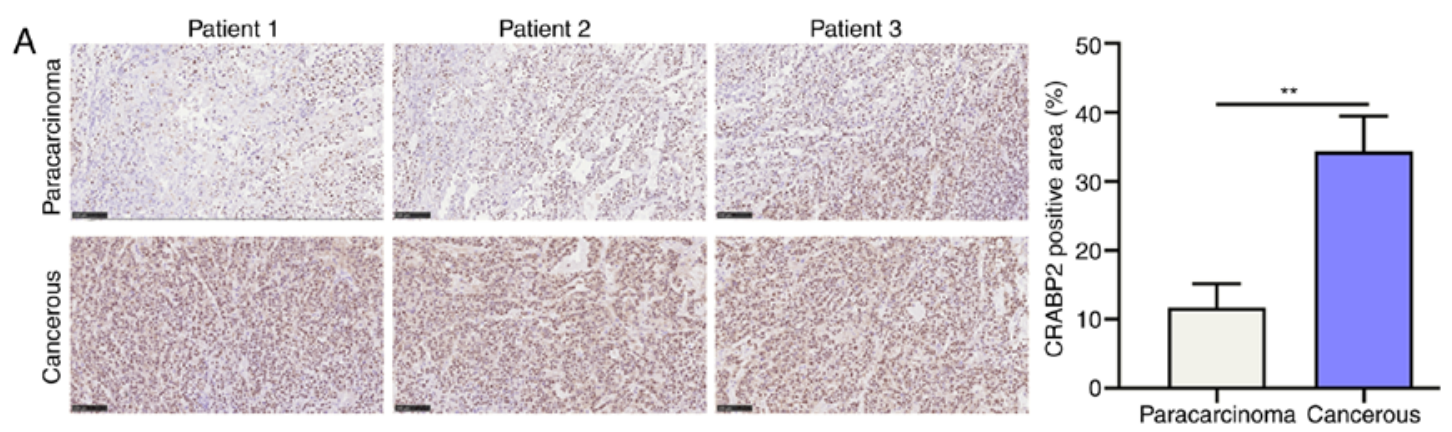

B
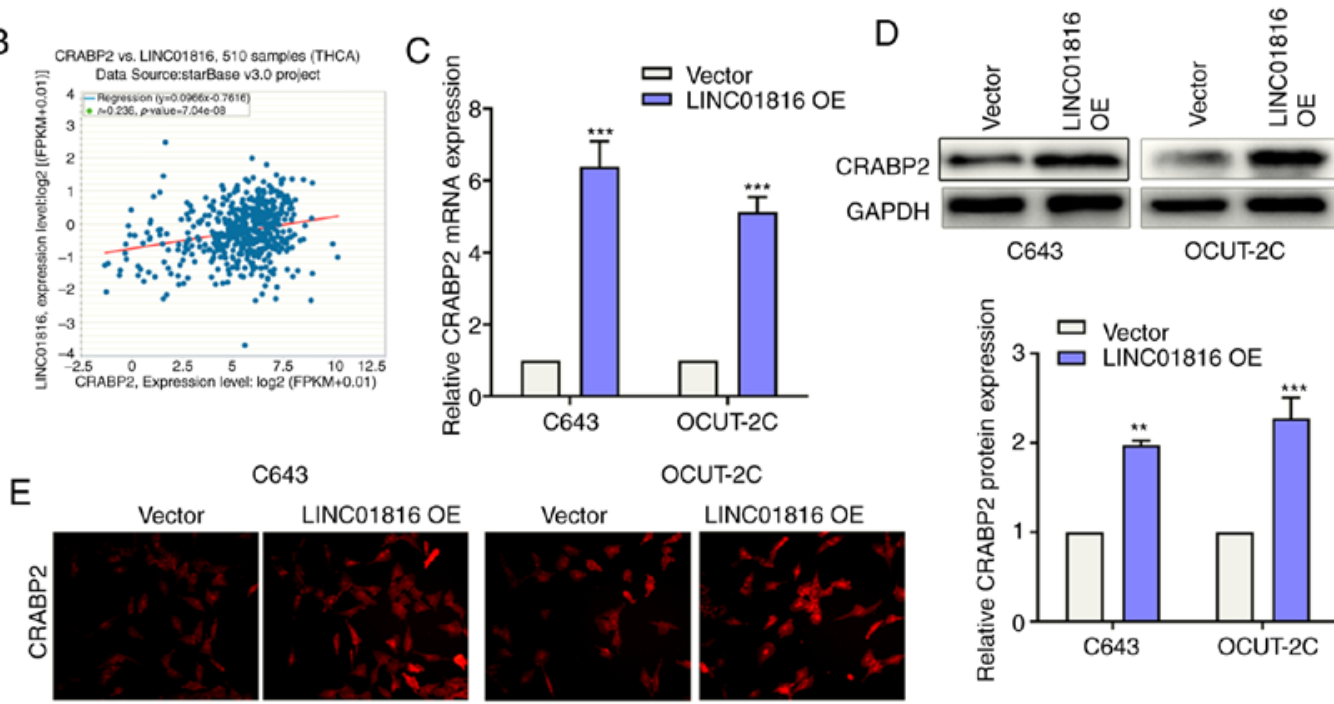

C643

OCUT-2C
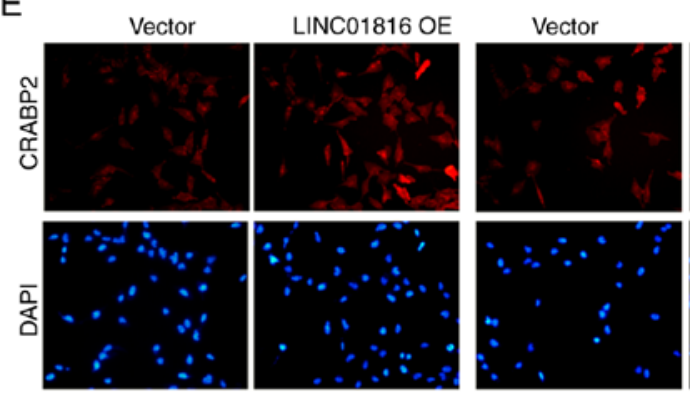

LINC01816 OE
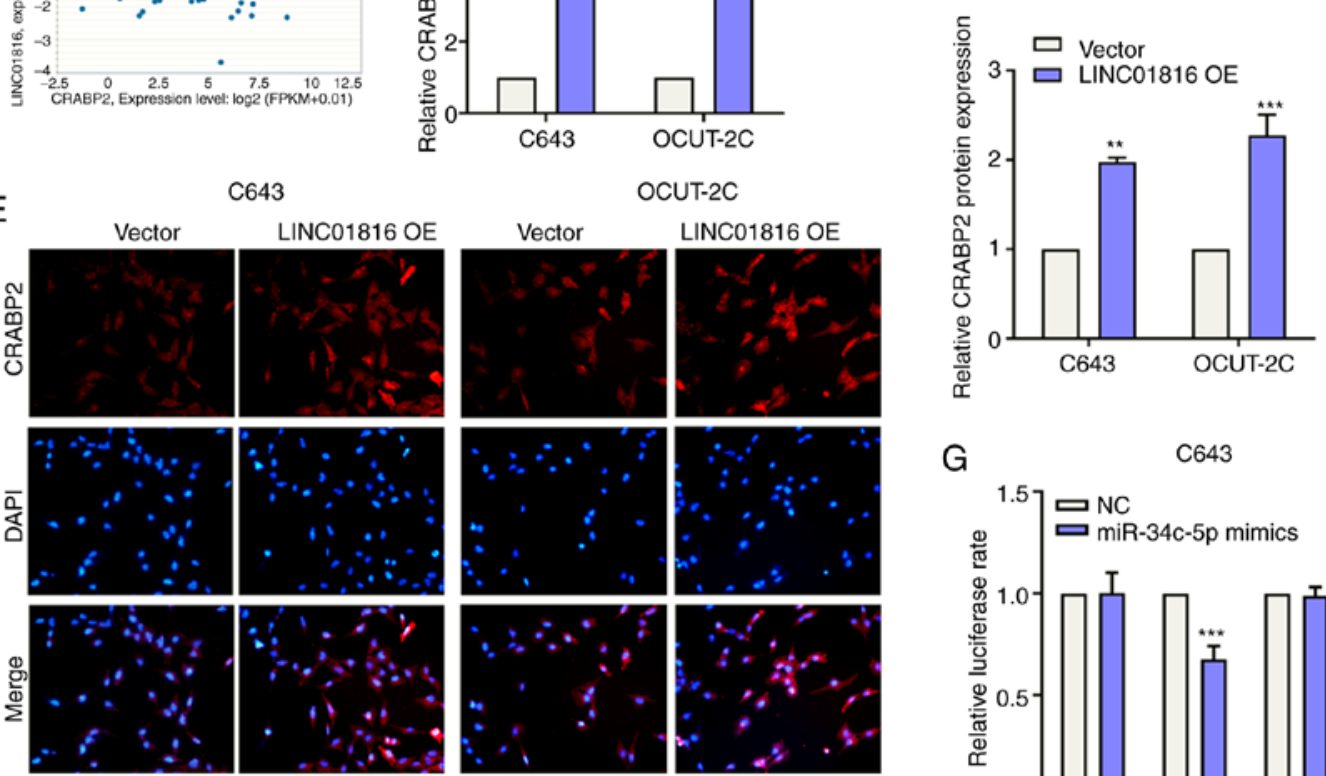

G

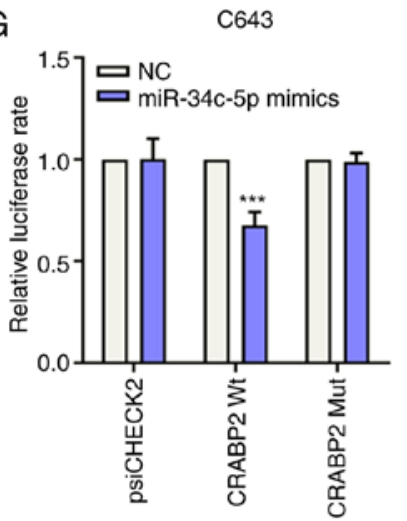

$\mathrm{F}$ $\begin{array}{cc}\text { Wt:CRABP2 } & \text { 3' CUCACCGCCCUGCUUCACUGCCC 5' } \\ \text { miR-34c-5p } & 3^{\prime} \text { CGUUAGUCGAUUGAUGUGACGGA } \\ \text { Mut:CRABP2 } & 3^{\prime} \text { CUCACCGCCCUGCUUGUGACGGC 5 }\end{array}$

$\mathrm{H}$

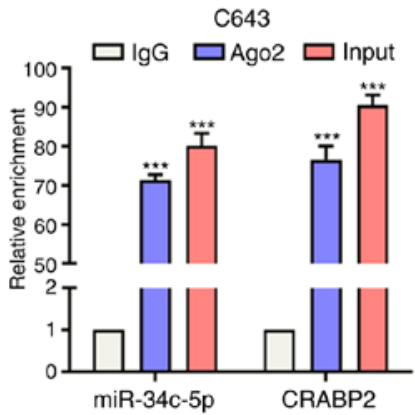

OCUT-2C

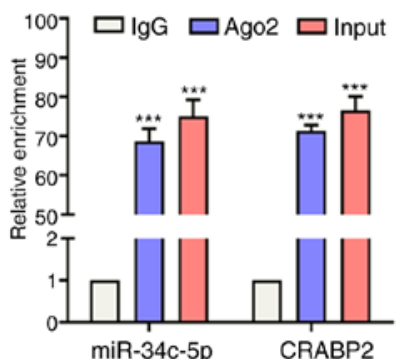

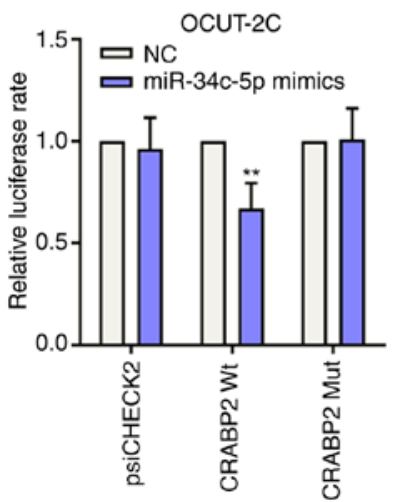

Figure 4. miR-34c-5p binds the 3 'UTR of CRABP2. (A) Immunohistochemical analysis of CRABP2 in cancerous (N=10) and paracarcinoma tissue (N=10). (B) The relationship between CRABP2 and LINC01816 from The Cancer Genome Atlas database. (C) The mRNA expression of CRABP2 was measured after LINC01816 overexpression in C643 and OCUT-2C cells. (D) The protein expression of CRABP2 was assessed after LINC01816 overexpression in C643 and OCUT-2C cells. (E) Immunofluorescence staining was used to detect CRABP2 expression after LINC01816 overexpression in C643 and OCUT-2C cells. (F) Schematic diagram of the miR-34c-5p putative binding sites and mutant site in the 3'UTR of CRABP2. (G) Dual luciferase reporter gene assay revealed that miR-34c-5p significantly decreased the luciferase activity of CRABP2 3'UTR. (H) RNA immunoprecipitation assay of the enrichment of Ago2 on CRABP2 relative to IgG in C643 and OCUT-2C cells. All of the aforementioned experiments were repeated at least three times. ${ }^{* *} \mathrm{P}<0.01$ and ${ }^{* * * *} \mathrm{P}<0.001$. miR-34c-5p, microRNA-34c-5p; CRABP2, cellular retinoic acid binding protein 2; LINC01816, long intergenic non-protein coding RNA 1816; OE, overexpression, NC, negative control; Wt, wild-type; Mut, mutant. 


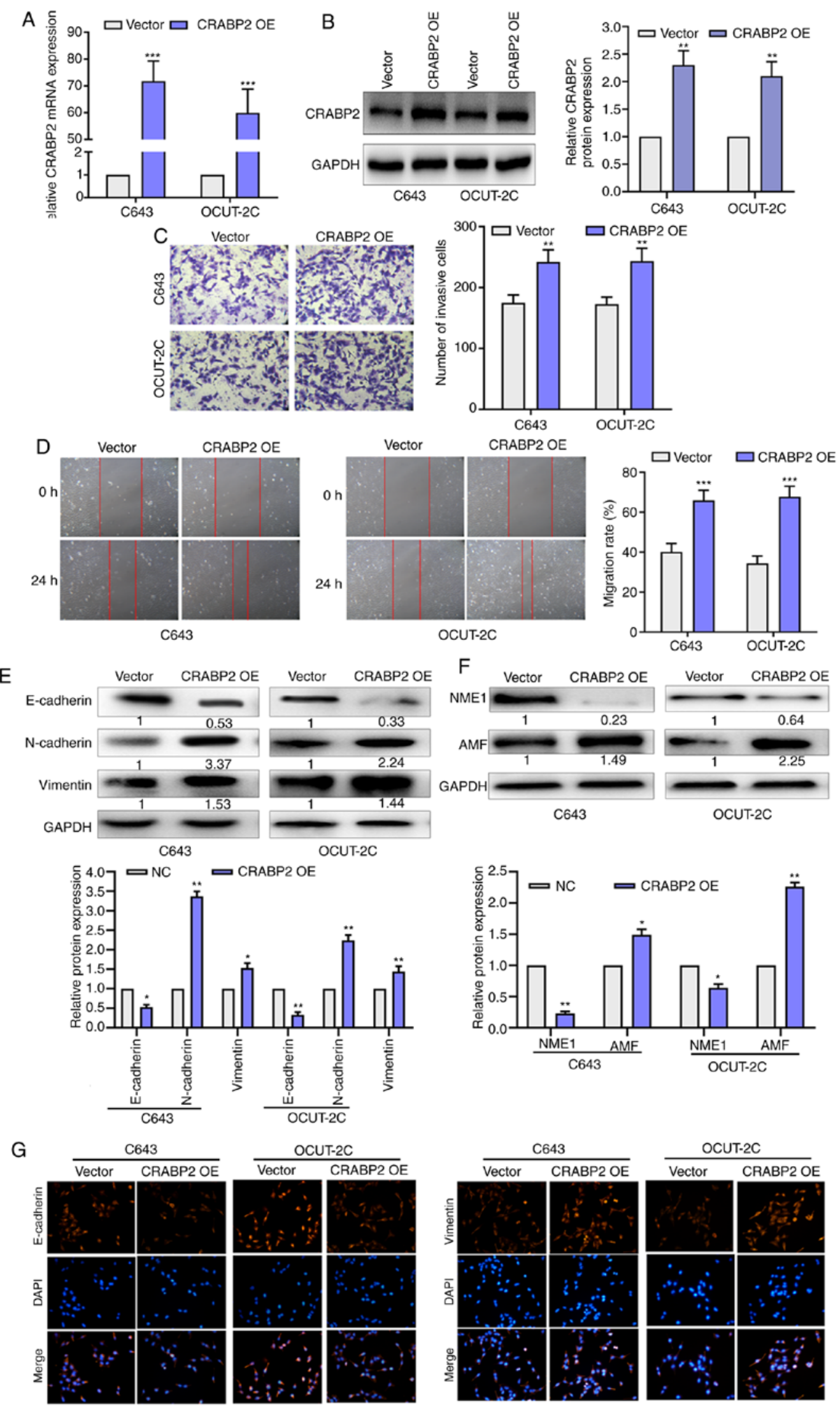

Figure 5. CRABP2 promotes EMT and cell migration. (A) The mRNA expression of CRABP2 was detected after CRABP2 overexpression in C643 and OCUT-2C cells. (B) The protein expression of CRABP2 was detected after CRABP2 overexpression in C643 and OCUT-2C cells. (C) Transwell assay was performed to assess the invasive ability of C643 and OCUT-2C cells with CRABP2 overexpression. (D) Scratch wound healing assay was performed to assess the migration ability of C643 and OCUT-2C cells with CRABP2 overexpression. (E) The EMT-related proteins were detected after CRABP2 overexpression in C643 and OCUT-2C. (F) The migration-related proteins were detected after CRABP2 overexpression in C643 and OCUT-2C cells. (G) Immunofluorescence staining was used to detect E-cadherin and vimentin expression after CRABP2 overexpression in C643 and OCUT-2C cells. All of the aforementioned experiments were repeated at least three times. ${ }^{*} \mathrm{P}<0.05,{ }^{* *} \mathrm{P}<0.01$ and ${ }^{* * * *} \mathrm{P}<0.001$. LINC01816, long intergenic non-protein coding RNA 1816; miR-34c-5p, microRNA-34c-5p; EMT, epithelial-mesenchymal transition; OE, overexpression; NC, negative control. 
Thyroid cancer vs Normal tissue

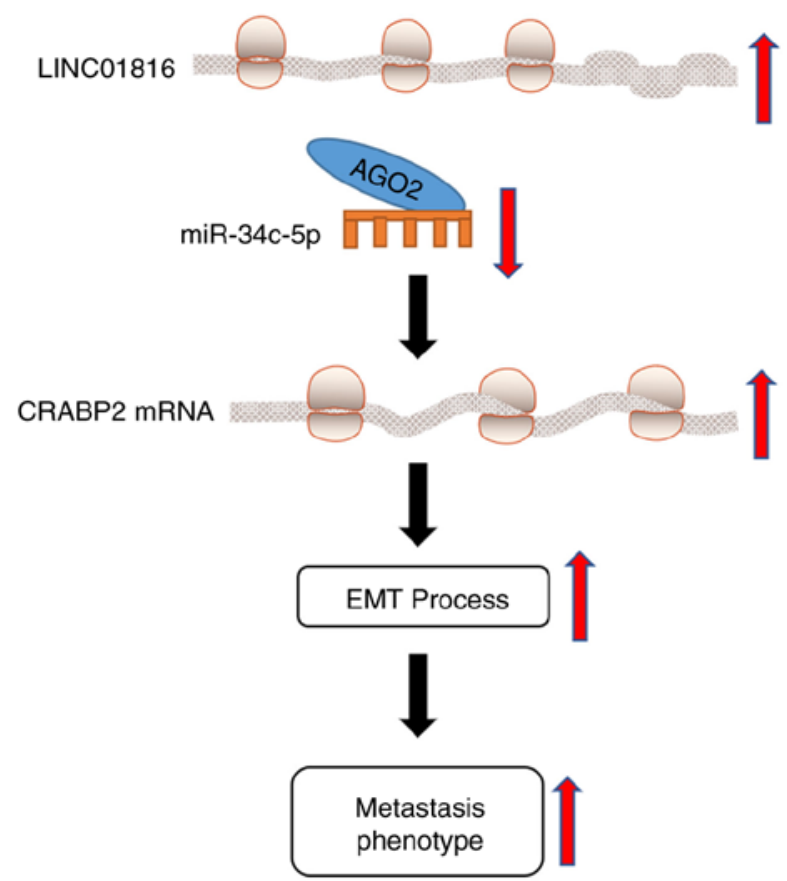

Figure 6. Schematic diagram of the proposed mechanism. LINC01816 promotes the migration, invasion and epithelial-mesenchymal transition of thyroid carcinoma cells by sponging miR-34c-5p and regulating CRABP2 expression levels. LINC01816, long intergenic non-protein coding RNA 1816; miR-34c-5p, microRNA-34c-5p; LINC01816, long intergenic non-protein coding RNA 1816; miR-34c-5p, microRNA-34c-5p; EMT, epithelialmesenchymal transition.

tissues. starBase was used to determine the presence of a positive association between CRABP2 and LINC01816 expression levels in THCA (Fig. 4B). Following the overexpression of LINC01816 in C643 and OCUT-2C cells, CRABP2 expression levels were revealed to be upregulated (Fig. 4C and D). The results of the immunofluorescence analysis also validated these findings, as the expression levels of CRABP2 were consistent with the results of the aforementioned western blotting (Fig. 4E). The binding site between CRABP2 and miR-34c-5p is presented in Fig. 4F. Subsequently, luciferase reporter plasmids containing 3'-UTR-CRABP2-Wt/Mut were constructed. The results of the dual-luciferase reporter assay demonstrated that the overexpression of miR-34c-5p suppressed the relative luciferase activity of the 3'-UTR-CRABP2-Wt vector, but not of the 3'-UTR-CRABP2-Mut vector, in C643 and OCUT-2C cells (Fig. 4G). The RIP assay results demonstrated that miR-34c-5p overexpression significantly increased the enrichment of CRABP2 in RIP-Ago2 compared with RIP-IgG in both C643 and OCUT-2C cells (Fig. 4H). In addition, other potential targets of miR-34c-5p were analyzed using the DAVID database. The related signaling pathways of miR-34c-5p target genes are presented in Fig. S4. miR-34c-5p was identified to be mainly involved in the following signaling pathways: 'Thyroid hormone signaling pathway' (44), 'pathway in cancer', 'MAPK signaling pathway' (45) and 'ErbB signaling pathway' (46), amongst others. These pathways are known to be closely associated with tumor processes. These results indicated that CRABP2 may be a target gene of miR-34c-5p.
The transfection efficiency of the CRABP2 overexpression plasmid was determined using RT-qPCR and western blotting. The mRNA and protein expression levels of CRABP2 were increased after CRABP2 overexpression plasmid transfection in C643 and OCUT-2C cells (Fig. 5A and B). As revealed in Fig. 5C, the number of invasive cells was increased in the CRABP2 overexpression group compared with the vector group. Wound healing assays were used to determine the role of CRABP2 in EMT progression. As presented in Fig. 5D, the migratory rate was increased in the cells overexpressing CRABP2 compared with the vector group. The protein expression levels of E-cadherin were downregulated in the CRABP2 overexpression group, while the expression levels of $\mathrm{N}$-cadherin and vimentin were upregulated in the CRABP2 overexpression group. The expression levels of NME1 were also downregulated following the overexpression of CRABP2, while AMF expression levels were upregulated in C643 and OCUT-2C cells (Fig. 5E and F). Immunofluorescence staining was used to further confirm these findings (Fig. 5G). The expression of E-cadherin was decreased while the expression of vimentin was increased with CRABP2 overexpression. These results indicated that the overexpression of LINC01816 induced the EMT process and led to an increase in cell migration. CRABP2 may be a target gene of miR-34c-5p and may be associated with EMT, migration and invasion in THCA.

\section{Discussion}

THCA has been classified as one of the fastest growing solid malignant tumors in China in the past 20 years (47). Although THCA has a low mortality rate, patients with advanced disease cannot be cured and thus, depend on drugs to maintain their quality of life (48). In the present study, analysis using TCGA database revealed that LINC01816 expression levels were significantly upregulated in THCA. In addition, LINC01816 knockdown could significantly suppress the migratory and invasive abilities of THCA. To clarify the mechanism of action of LINC01816 in the regulation of THCA progression, bioinformatics analysis was first used, and the results were subsequently verified using in vitro cell line experiments. The results demonstrated that miR-34c-5p shared a complementary binding site with LINC01816 and its expression levels were regulated by LINC01816 in vitro. LncRNAs are located in the cytoplasm, where they can sponge miRNAs to regulate gene expression (49). Previous studies have reported that miR-34c-5p was involved in regulating tumor progression. For example, miR-34c-5p inhibited the stemness of ovarian cancer and the development of drug resistance by downregulating the amphiregulin/EGFR/ERK signaling pathway (50). Wang et al determined that miR-34c-5p targeted flotillin 2 to inhibit the proliferation, migration and invasion of osteosarcoma cells, downregulate the protein expression levels of cyclin D1, matrix metalloproteinase (MMP)-2 and MMP-9, and upregulate the protein expression levels of p21 (51). The signaling pathways associated with miR-34c-5p target genes are known to be closely associated with tumor processes (23-25). The results of the present study also revealed that E-cadherin expression levels were significantly downregulated following the inhibition of miR-34c-5p. In addition, the transfection of 
THCA cells with miR-34c-5p mimics reduced the migratory ability. These data indicated that miR-34c-5p may serve as a tumor suppressor in the regulation of tumor progression. In addition, the overexpression of LINC01816 could reverse the inhibitory effect of miR-34c-5p overexpression on THCA progression. Thus, these findings indicated that LINC01816 may be involved in the regulation of THCA as an oncogenic factor. Notably, the knockdown of miR-34c-5p enhanced the effects of LINC01816.

CRABP2 is a cellular lipid-binding protein that is involved in the transport of retinoic acid (52). The aberrant regulation of CRABP2 is a common phenomenon in numerous different cancer types. CRABP2 expression levels were reported to be downregulated in prostate cancer, esophageal squamous cell carcinoma, head and neck squamous cell carcinoma and astrocytic glioma, amongst others. In contrast, the upregulation of CRABP2 expression levels has been reported in retinoblastoma, ovarian cancer, breast cancer, lung cancer and uterine leiomyoma (53-56). However, to the best of our knowledge, little is known about the role of CRABP 2 in THCA. The results of the present study revealed that the genetic knockdown of CRABP2 suppressed cell migration, invasion and EMT in C643 and OCUT-2C cells. In addition, the expression levels of NME1 were revealed to be downregulated following the overexpression of CRABP2. Further experiments verified that CRABP2 expression levels were positively associated with LINC01816 expression levels, and its expression was regulated by LINC01816 and miR-34c-5p.

Collectively, the findings of the present study provided significant evidence to suggest that LINC01816 expression levels may be significantly upregulated in cancer tissues compared with matched adjacent tissues. In addition, according to TCGA database analysis, LINC01816 expression levels were determined to be modulated by the miR-34c-5p/CRABP2 ceRNA regulatory network. However, there are also limitations to the present study. For example, the results were not verified using an in vivo animal model or an independent cohort of patients. Therefore, these issues will be addressed in future studies.

In conclusion, the findings of the present study proposed a novel molecular mechanism for LINC01816 in regulating the process of EMT in THCA. The results revealed that LINC01816 expression levels were upregulated in THCA, which may subsequently serve a role in the migratory and EMT processes of THCA cells by targeting the miR-34c-5p/CRABP 2 axis (Fig. 6). Therefore, the LINC01816/miR-34c-5p/CRABP2 axis may be of clinical significance in THCA.

\section{Acknowledgements}

Not applicable.

\section{Funding}

No funding was received.

\section{Availability of data and material}

All data generated or analyzed during this study are included in this published article.

\section{Author's contributions}

HZ contributed to the design of the study, wrote the manuscript and analyzed the data. XZ and YL revised the manuscript and contributed to the design of the study. SL, WW and LZ acquired, analyzed and interpreted the data. JZ made substantial contributions to the conception and design of the present study and revised the manuscript. All authors read and approved the final manuscript.

\section{Ethics approval and consent to participate}

This present study was approved by the Ethics Committee of the Affiliated Hospital of Zunyi Medical University (Zunyi, China) and written informed consent was obtained from all patients.

\section{Patient consent for publication}

Not applicable.

\section{Competing interests}

The authors declare that they have no competing interests.

\section{References}

1. Ciavardelli D, Bellomo M, Consalvo A, Crescimanno C and Vella V: Metabolic alterations of thyroid cancer as potential therapeutic targets. Biomed Res Int 2017: 2545031, 2017.

2. Vaccarella S, Franceschi S, Bray F, Wild CP, Plummer M and Dal Maso L: Worldwide thyroid-cancer epidemic? The increasing impact of overdiagnosis. N Engl J Med 375: 614-617, 2016.

3. Sanabria A, Kowalski LP, Shah JP, Nixon IJ, Angelos P, Williams MD, Rinaldo A and Ferlito A: Growing incidence of thyroid carcinoma in recent years: Factors underlying overdiagnosis. Head Neck 40: 855-866, 2018.

4. Haramati S, Chapnik E, Sztainberg Y, Eilam R, Zwang R, Gershoni N, McGlinn E, Heiser PW, Wills AM, Wirguin I, et al: miRNA malfunction causes spinal motor neuron disease. Proc Natl Acad Sci USA 107: 13111-13116, 2010.

5. Thomson DW and Dinger ME: Endogenous microRNA sponges: Evidence and controversy. Nat Rev Genet 17: 272-283, 2016.

6. Lacouture ME, Ciccolini K, Kloos RT and Agulnik M: Overview and management of dermatologic events associated with targeted therapies for medullary thyroid cancer. Thyroid 24: 1329-1340, 2014.

7. Kim H, Kim YN, Kim HI, Park SY, Choe JH, Kim JH, Kim JS, Chung JH, Kim TH and Kim SW: Preoperative serum thyroglobulin predicts initial distant metastasis in patients with differentiated thyroid cancer. Sci Rep 7: 16955, 2017.

8. Wang M, Qiu S and Qin J: Baicalein induced apoptosis and autophagy of undifferentiated thyroid cancer cells by the ERK/PI3K/Akt pathway. Am J Transl Res 11: 3341-3352, 2019.

9. Jung CW, Han KH, Seol H, et al: Expression of cancer stem cell markers and epithelial-mesenchymal transition-related factors in anaplastic thyroid carcinoma. International journal of clinical and experimental pathology 8: 560-568, 2015.

10. Sandberg K, Samson WK and Ji H: Decoding noncoding RNA: da Vinci redux? Circ Res 113: 240-241, 2013.

11. Diamantopoulos MA, Tsiakanikas P and Scorilas A: Non-coding RNAs: The riddle of the transcriptome and their perspectives in cancer. Ann Transl Med 6: 241, 2018.

12. Dai M, Li S and Qin X: Colorectal neoplasia differentially expressed: A long noncoding RNA with an imperative role in cancer. Onco Targets Ther 11: 3755-3763, 2018.

13. Ogoyama M, Ohkuchi A, Takahashi H, Zhao D, Matsubara S and Takizawa T: LncRNA H19-derived miR-675-5p accelerates the invasion of extravillous trophoblast cells by inhibiting GATA2 and subsequently activating matrix metalloproteinases. Int J Mol Sci 22: 1237, 2021. 
14. Iorio MV and Croce CM: MicroRNA dysregulation in cancer: Diagnostics, monitoring and therapeutics. A comprehensive review. EMBO Mol Med 4: 143-159, 2012.

15. Muhammad N, Bhattacharya S, Steele R and Ray RB Anti-miR-203 suppresses ER-positive breast cancer growth and stemness by targeting SOCS3. Oncotarget 7: 58595-58605, 2016.

16. Yanaihara $\mathrm{N}$ and Harris CC: MicroRNA involvement in human cancers. Clin Chem 59: 1811-1812, 2013.

17. Rosignolo F, Memeo L, Monzani F, Colarossi C, Pecce V, Verrienti A, Durante C, Grani G, Lamartina L, Forte S, et al: MicroRNA-based molecular classification of papillary thyroid carcinoma. Int J Oncol 50: 1767-1777, 2017.

18. Dong S, Xue S, Sun Y, Han Z, Sun L, Xu J and Liu J: MicroRNA-363-3p downregulation in papillary thyroid cancer inhibits tumor progression by targeting NOB1. J Investig Med 69: 66-74, 2021.

19. Liu HM, Dong AB, Hua H, Sun YH, Wang JR, Yu QQ, Zhang JH and Sun WH: MicroRNA-219 inhibits cell viability and metastasis in papillary thyroid carcinoma by targeting EYA2. Eur Rev Med Pharmacol Sci 24: 9556-9564, 2020.

20. Li N, Mao D, Cao Y, Li H, Ren F and Li K: Downregulation of SIRT6 by miR-34c-5p is associated with poor prognosis and promotes colon cancer proliferation through inhibiting apoptosis via the JAK2/STAT3 signaling pathway. Int J Oncol 52: 1515-1527, 2018.

21. Liang M, Yu S, Tang S, Bai L, Cheng J, Gu Y, Li S, Zheng X, Duan L, Wang L, et al: A panel of plasma exosomal mirnas as potential biomarkers for differential diagnosis of thyroid nodules. Front Genet 11: 449, 2020.

22. Shen Y, Xu J, Pan X, Zhang Y, Weng Y, Zhou D and He S: LncRNA KCNQ1OT1 sponges miR-34c-5p to promote osteosarcoma growth via ALDOA enhanced aerobic glycolysis. Cell Death Dis 11: 278, 2020.

23. Yanokura M, Banno K and Aoki D: MicroRNA-34b expression enhances chemosensitivity of endometrial cancer cells to paclitaxel. Int J Oncol 57: 1145-1156, 2020.

24. Lin BZ, Wan SY, Lin MY, Chang CH, Chen TW, Yang MH and Lee YJ: Involvement of differentially expressed microRNAs in the PEGylated liposome encapsulated ${ }^{188}$ rhenium-mediated suppression of orthotopic hypopharyngeal tumor. Molecules 25: 3609, 2020

25. Wang H, Bian S and Yang CS: Green tea polyphenol EGCG suppresses lung cancer cell grow th through upregulating miR-210 expression caused by stabilizing HIF-1 $\alpha$. Carcinogenesis 32: 1881-1889, 2011

26. Kazimierczyk M, Kasprowicz MK, Kasprzyk ME and Wrzesinski J: Human long noncoding RNA interactome: detection, characterization and function. Int J Mol Sci 21: 1027, 2020.

27. Salmena L, Poliseno L, Tay Y, Kats L and Pandolfi PP: A ceRNA hypothesis: The Rosetta Stone of a hidden RNA language? Cell 146: 353-358, 2011.

28. Fan H, Ge Y, Ma X, Li Z, Shi L, Lin L, Xiao J, Chen W, Ni P, Yang L and Xu Z: Long non-coding RNA CCDC144NL-AS1 sponges miR-143-3p and regulates MAP3K7 by acting as a competing endogenous RNA in gastric cancer. Cell Death Dis 11: $521,2020$.

29. Yu H, Zhong X, Gao P, Shi J, Wu Z, Guo Z, Wang Z and Song Y: The potential effect of metformin on cancer: An umbrella review. Front Endocrinol (Lausanne) 10: 617, 2019.

30. Bayat Mokhtari R, Homayouni TS, Baluch N, Morgatskaya E, Kumar S, Das B and Yeger H: Combination therapy in combating cancer. Oncotarget 8: 38022-38043, 2017.

31. Muhammad N, Steele R, Isbell TS, Philips N and Ray RB: Bitter melon extract inhibits breast cancer growth in preclinical model by inducing autophagic cell death. Oncotarget 8: 66226-66236, 2017.

32. Mohammad N, Malvi P, Meena AS, Singh SV, Chaube B Vannuruswamy G, Kulkarni MJ and Bhat MK: Cholesterol depletion by methyl- $\beta$-cyclodextrin augments tamoxifen induced cell death by enhancing its uptake in melanoma. Mol Cancer 13: 204, 2014.

33. Mohammad N, Singh SV, Malvi P, Chaube B, Athavale D, Vanuopadath M, Nair SS, Nair B and Bhat MK: Strategy to enhance efficacy of doxorubicin in solid tumor cells by methyl- $\beta$-cyclodextrin: Involvement of p53 and Fas receptor ligand complex. Sci Rep 5: 11853, 2015.

34. Gupta A, Williams BR, Hanash SM and Rawwas J: Cellular retinoic acid-binding protein II is a direct transcriptional target of MycN in neuroblastoma. Cancer Res 66: 8100-8108, 2006.

35. Gupta A, Kessler P, Rawwas J and Williams BR: Regulation of CRABP-II expression by $\mathrm{MycN}$ in Wilms tumor. Exp Cell Res 314: 3663-3668, 2008.
36. Favorskaya I, Kainov Y, Chemeris G, Komelkov A, Zborovskaya I and Tchevkina E: Expression and clinical significance of CRABP1 and CRABP2 in non-small cell lung cancer. Tumour Biol: 35: 10295-10300, 2014.

37. Donato LJ and Noy N: Suppression of mammary carcinoma growth by retinoic acid: Proapoptotic genes are targets for retinoic acid receptor and cellular retinoic acid-binding protein II signaling. Cancer Res 65: 8193-8199, 2005.

38. Budhu AS and Noy N: Direct channeling of retinoic acid between cellular retinoic acid-binding protein II and retinoic acid receptor sensitizes mammary carcinoma cells to retinoic acid-induced growth arrest. Mol Cell Biol 22: 2632-2641, 2002.

39. Livak KJ and Schmittgen TD: Analysis of relative gene expression data using real-time quantitative PCR and the 2(-Delta Delta C(T)) method. Methods 25: 402-408, 2001.

40. Tang Z, Li C, Kang B, Gao G, Li C and Zhang Z: GEPIA: A web server for cancer and normal gene expression profiling and interactive analyses. Nucleic Acids Res 45: W98-W102, 2017.

41. Li JH, Liu S, Zhou H, Qu LH and Yang JH: starBase v2.0: Decoding miRNA-ceRNA, miRNA-ncRNA and protein-RNA interaction networks from large-scale CLIP-Seq data. Nucleic Acids Res 42 (Database Issue): D92-D97, 2013.

42. Agarwal V, Bell GW, Nam JW and Bartel DP: Predicting effective microRNA target sites in mammalian mRNAs. Elife 4 : $\mathrm{e} 05005,2015$.

43. Jiao X, Sherman BT, Huang da W, Stephens R, Baseler MW, Lane HC and Lempicki RA: DAVID-WS: A stateful web service to facilitate gene/protein list analysis. Bioinformatics 28 : 1805-1806, 2012

44. Jonklaas J, Nsouli-Maktabi H and Soldin SJ: Endogenous thyrotropin and triiodothyronine concentrations in individuals with thyroid cancer. Thyroid 18: 943-952, 2008.

45. Jiang W, Cai F, Xu H, Lu Y, Chen J, Liu J, Cao N, Zhang X, Chen X, Huang Q, et al: Extracellular signal regulated kinase 5 promotes cell migration, invasion and lung metastasis in a FAK-dependent manner. Protein Cell 11: 825-845, 2020.

46. Mahtouk K, Jourdan M, De Vos J, Hertogh C, Fiol G, Jourdan E, Rossi JF and Klein B: An inhibitor of the EGF receptor family blocks myeloma cell growth factor activity of HB-EGF and potentiates dexamethasone or anti-IL-6 antibody-induced apoptosis. Blood 103: 1829-1837, 2004.

47. Brito JP, Morris JC and Montori VM: Thyroid cancer: Zealous imaging has increased detection and treatment of low risk tumours. BMJ 347: f4706, 2013.

48. Lirov R, Worden FP and Cohen MS: The treatment of advanced thyroid cancer in the age of novel targeted therapies. Drugs 77: 733-745, 2017.

49. Rashid F, Shah A and Shan G: Long non-coding RNAs in the cytoplasm. Genomics Proteomics Bioinformatics 14: 73-80, 2016.

50. Tung SL, Huang WC, Hsu FC, Yang ZP, Jang TH, Chang JW, Chuang CM, Lai CR and Wang LH: miRNA-34c-5p inhibits amphiregulin-induced ovarian cancer stemness and drug resistance via downregulation of the AREG-EGFR-ERK pathway. Oncogenesis 6: e326, 2017.

51. Wang Y, Wang X, Tang J, Su X and Miao Y: The study of mechanism of miR-34c-5p targeting FLOT2 to regulate proliferation, migration and invasion of osteosarcoma cells. Artif Cells Nanomed Biotechnol 47: 3559-3568, 2019.

52. Yuan J, Tang Z, Yang S and Li K: CRABP2 promotes myoblast differentiation and is modulated by the transcription factors MyoD and Sp1 in C2C12 cells. PLoS One 8: e55479, 2013.

53. Okuducu AF, Janzen V, Ko Y, Hahne JC, Lu H, Ma ZL, Albers P, Sahin A, Wellmann A, Scheinert P and Wernert N: Cellular retinoic acid-binding protein 2 is down-regulated in prostate cancer. Int J Oncol 27: 1273-1282, 2005.

54. Wu JI, Lin YP, Tseng CW, Chen HJ and Wang LH: Crabp2 promotes metastasis of lung cancer cells via HuR and integrin $\beta 1 / F A K / E R K$ signaling. Sci Rep 9: 845, 2019.

55. Li YT, Tian XT, Wu ML, Zheng X, Kong QY, Cheng XX, Zhu GW, Liu J and Li H: Resveratrol suppresses the growth and enhances retinoic acid sensitivity of anaplastic thyroid cancer cells. Int J Mol Sci 19: 1030, 2018.

56. Liu RZ, Li S, Garcia E, Glubrecht DD, Poon HY, Easaw JC and Godbout R: Association between cytoplasmic CRABP2, altered retinoic acid signaling, and poor prognosis in glioblastoma. Glia 64: 963-976, 2016.

This work is licensed under a Creative Commons Attribution-NonCommercial-NoDerivatives 4.0 International (CC BY-NC-ND 4.0) License. 